\title{
Study of far-end crosstalk between LTCC microstrip lines
}

\author{
Yuming $\mathrm{Wu}^{1, \mathrm{a}}$, Zhaohua $\mathrm{Wu}^{1, \mathrm{~b}}$ \\ ${ }^{1}$ School of Electromechanical Engineering, Guilin University of Electronic Technology, Guilin, \\ 541004, China \\ a1511528155@qq.com, bemezdj@guet.edu.cn
}

Keywords: far-end crosstalk; LTCC microstrip lines; air-cavity

\begin{abstract}
In order to reduce far-end crosstalk between LTCC microstrip lines, this paper proposes a novel structure that has an air-cavity in dielectric layer. Simulation model of LTCC microstrip lines was set up by using Ansoft HFSS, the far-end crosstalk $\left(\mathrm{S}_{14}\right)$ was obtained, the effects of cavity height, cavity width, location of cavity on far-end crosstalk strength between lines were studied. The results show that far-end crosstalk of proposed structure is reduced more than $12 \mathrm{~dB}$ from 18 $\mathrm{GHz}$ to $25.8 \mathrm{GHz}$ compared with taking no any measures. The effect of reducing far-end crosstalk is nonlinear increasing with increasing of cavity width; the effect of reducing far-end crosstalk is increasing and then decreasing with increasing of cavity height. However, cavity height of the effect best is different in different frequency, in contrast, cavity height of the effect best is $0.14 \mathrm{~mm}$. When location of sample cavity in dielectric layer is different, the effect of reducing far-end crosstalk is different. Distance from bottom of cavity to ground plane of the effect best is $0.06 \mathrm{~mm}$.
\end{abstract}

\section{Introduction}

With the rapid development of the millimeter-wave technology, the requirement of miniaturization, low cost, reliability and volume production of equipments and products is increasing[1,2]. In millimeter-wave integrated circuits, crosstalk occurs in the transmission lines of high density, because of edge-field[3]. Many scholars adopt various methods to reduce far-end crosstalk between microstrip lines. One of the most commonly used methods is to join different shapes of guard lines between microstrip lines. For the no guard, the conventional guard, the via-stitch guard, and the serpentine guard, among these four cases, the serpentine guard gives the smallest values in both the peak far-end crosstalk voltage and the timing jitter. [4]. Method of the metal-filled via holes is used to improve the effect of reducing far-end crosstalk from $25 \mathrm{GHz}$ to 45 $\mathrm{GHz}[2]$. Lin proposes a method to reduce far-end crosstalk by using rectangular-shape resonators (RSR) structure, gets a better result that the $\mathrm{S}_{41}$ of RSR structure is decreased more than $7 \mathrm{~dB}$ compared to the $3-\mathrm{W}$ rule[5]. In the certain frequency range, it is an effective way to suppress far-end crosstalk in large scale by increasing the length and number of metallic sheets; the method of selecting large relative permittivity and thickness of media layer can also reduce the effect of far-end crosstalk[6].

The inductor with an air-gap show increase of the quality factor over 30 percent and widening of the operating frequency range by 50 percent when compared with the same LTCC structure without a gap[7]. So this paper proposes a novel structure that has an air-cavity in dielectric layer to reduce far-end crosstalk between LTCC microstrip lines. Simulation model of LTCC microstrip lines is set up by using Ansoft HFSS, for the no guard, the metal-filled via holes, RSR structure, structure proposed ,among these four cases, we discuss the effect of reducing far-end crosstalk by comparing S41, then the effect of cavity height, cavity width, location of cavity on far-end crosstalk strength between lines are studied.

\section{The calculation of S parameter}

$\mathrm{S}$ parameter is based on the relationship between incident wave and reflected wave of network parameters, as shown in figure 1 for $\mathrm{N}$ ports network diagram. Incident power and reflection power associated with the port I can be defined as [8]: 


$$
\begin{aligned}
& a_{i}=\frac{V_{i}+Z_{i} I_{i}}{2 \sqrt{\left|\operatorname{Re} Z_{i}\right|}} \\
& b_{i}=\frac{V_{i}-Z_{i} I_{i}}{2 \sqrt{\left|\operatorname{Re} Z_{i}\right|}}
\end{aligned}
$$

Among them, $V_{i}$ and $I_{i}$ are respectively port voltage and current of flowing into, $Z_{i}$ is impedance looked out from port, $Z^{*}{ }_{i}$ is complex conjugate of ${ }^{Z_{i}}$.

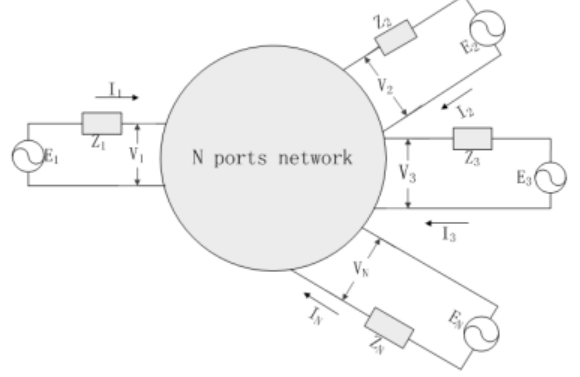

Fig. 1 diagram of $\mathrm{N}$ ports network

In microwave circuits, $Z_{i}$ is usually a real number and equal to $50 \Omega$. therefore, the $\mathrm{S}$ parameter matrix can be written as:

$$
\left[\begin{array}{l}
b_{1} \\
b_{2} \\
\cdots \\
b_{N}
\end{array}\right]=\left[\begin{array}{llll}
S_{11} & S_{12} & \ldots & S_{1 N} \\
S_{21} & S_{22} & \ldots & S_{2 N} \\
\ldots & \ldots & \ldots & \ldots \\
S_{N 1} & S_{N 2} & \ldots & s_{N N}
\end{array}\right] \times\left[\begin{array}{c}
a_{1} \\
a_{2} \\
\ldots \\
a_{N}
\end{array}\right]
$$

${ }^{S}$ is is used to define related scattering port, $\mathrm{m}$ will be output port, $\mathrm{n}$ will be input port. When only $\mathrm{n}$ port is incentive port and other ports are matching load, $b_{m}$ : the output power of output port, ${ }_{n}$ : the input power of input port, there is

$$
S_{m n}=\frac{b_{m}}{a_{n}}
$$

$S_{41}=\frac{b_{4}}{a_{1}}$
Therefore,

\section{Simulation model}
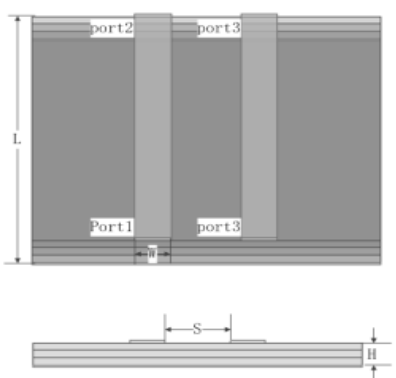

Fig. 2 the structure model of coupled microstrip lines

Figure 2 shows the structural model of two parallel microstrip lines, the substrate dielectric material is FERRO-A6, the relative dielectric constant of 5.9, dissipation factor of 0.002 , the dielectric layer thickness of $0.3 \mathrm{~mm}$, Single porcelain with $0.1 \mathrm{~mm}$ thickness; line thickness $\mathrm{T}$ as $0.035 \mathrm{~mm}$, line width $\mathrm{W}$ as $0.42 \mathrm{~mm}$, line length $\mathrm{L}$ as $10 \mathrm{~mm}$. 


\section{Methods of reducing far-end crosstalk}

At present, there are many methods of reducing far-end crosstalk between LTCC microstrip lines, for example adding distance between lines, adding guard lines and so on. It is a very effective method to add distance between lines. However it is difficult to implement with the requirement of miniaturization. This method will not be discussed. For the no guard, the guard with metal-filled via holes(distance between lines $\mathrm{G}$ as $0.7 \mathrm{~mm}$ in the model, distance between holes $\mathrm{S}$ as $0.25 \mathrm{~mm}$, hole radius $\mathrm{R}$ as $0.1 \mathrm{~mm}$ )[2], RSR structure (metal patch is made of copper, the number $\mathrm{n}$ as $7, \mathrm{~m}=0.5$ $\mathrm{mm}, \mathrm{dm}=1.5 \mathrm{~mm}$ )[6], structure proposed, figure 3 shows the effect of reducing far-end crosstalk among these four cases. The conclusion is obtained that structure proposed has better effect on reducing the far-end crosstalk from $18 \mathrm{GHz}$ to $25.8 \mathrm{GHz}$, compared with the guard lines with metal-filled via holes and RSR structure.

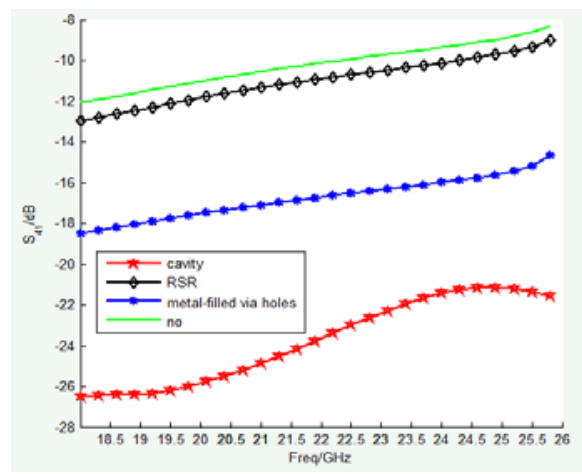

Fig. 3 the comparison of three measures of reducing far-end crosstalk

\section{Study of structure with the cavity}
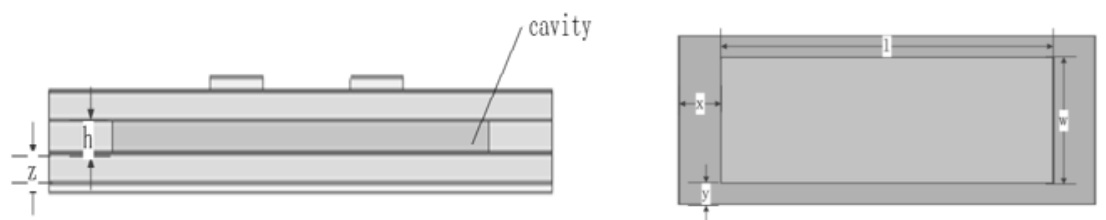

(a) cutaway view of 3D model (vertical) (b) cutaway view of 3D model (horizontal)

Fig. 4 diagram of structure with the cavity

Figure 4 shows structure with the cavity. Distance from left edge of the cavity to left edge of the medium layer is expressed by $\mathrm{x}$; distance from lower edge of the cavity to lower edge of the dielectric layer is expressed by $y$; distance from bottom of the cavity to the ground plane is expressed by z. Cavity length, width and height are separately expressed by I, w, h. For study of the effect of reducing far-end crosstalk by changing structure parameters of the cavity, $x=1 \mathrm{~mm}, \mathrm{y}=1$ $\mathrm{mm}, \mathrm{z}=0.1 \mathrm{~mm}, \mathrm{l}=8 \mathrm{~mm}, \mathrm{w}=3 \mathrm{~mm}$ are selected, then $\mathrm{h}$ is changed. Figure 5 shows $\mathrm{S} 41$ under different $h$. The conclusions are obtained from figure 5 . With increasing of cavity height, the effect of reducing far-end crosstalk is increasing and then decreasing from $18 \mathrm{GHz}$ to $25.8 \mathrm{GHz}$. The effect of reducing far-end crosstalk is similar in the structure of $h=0.14 \mathrm{~mm}$ and the structure of $\mathrm{h}=$ $0.16 \mathrm{~mm}$. In contrast, cavity height of effect best was $0.14 \mathrm{~mm}$ from 18 to $25.8 \mathrm{GHz}$.

When the relationship between the effect of reducing far-end crosstalk and location of the cavity in dielectric layer is discussed, $x=1 \mathrm{~mm}, \mathrm{y}=1 \mathrm{~mm}, \mathrm{l}=8 \mathrm{~mm}, \mathrm{w}=3 \mathrm{~mm}, \mathrm{~h}=0.14$ are selected based on those above conclusions. Then, $\mathrm{z}$ is changed. Figure 6 shows S41 under different $\mathrm{z}$. The conclusions are obtained from figure 6 . With increasing of $\mathrm{z}$, the effect of reducing far-end crosstalk is increasing and then decreasing from $18 \mathrm{GHz}$ to $25.8 \mathrm{GHz}$. Structure that has optimal effect is $\mathrm{z}=$ $0.06 \mathrm{~mm}$, compared with other four kinds of structures. Closer to microstrip line, the effect gets worse. The effect of reducing far-end crosstalk is similar in the structure of $\mathrm{z}=0.04 \mathrm{~mm}$ and the structure of $\mathrm{z}=0.08 \mathrm{~mm}$. 


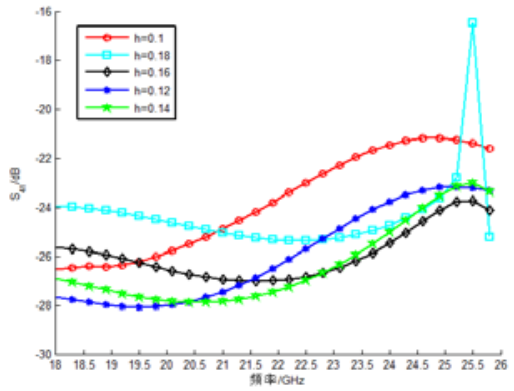

Fig. $5 \mathrm{~S}_{41}$ under different $\mathrm{h}$

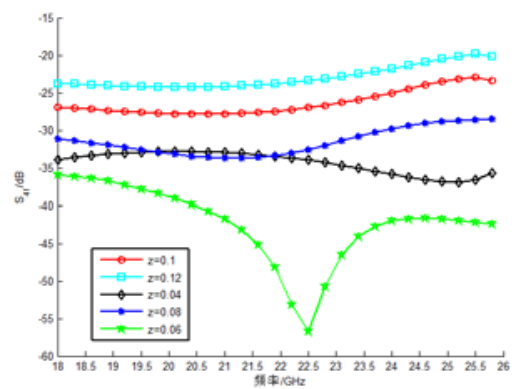

Fig. $6 \mathrm{~S}_{41}$ under different $\mathrm{z}$

When the relationship between the effect of reducing far-end crosstalk and cavity width is discussed, $\mathrm{x}=1 \mathrm{~mm}, \mathrm{y}=0.5 \mathrm{~mm}, \mathrm{z}=0.1 \mathrm{~mm}, \mathrm{l}=8 \mathrm{~mm}, \mathrm{~h}=0.1$ are selected. Then, $\mathrm{w}$ is changed. Figure 7 shows S41 under different w. Effect factors of reducing far-end crosstalk that cavity width and distance from left edge of the cavity to microstrip line in horizontal are found based on theoretical analysis, therefore the relationship between the effect of reducing far-end crosstalk and distance from left edges of the cavity to microstrip line in horizontal need to be discussed. $\mathrm{x}=1 \mathrm{~mm}, \mathrm{z}=0.1 \mathrm{~mm}$, $\mathrm{l}=8 \mathrm{~mm}, \mathrm{~h}=0.1 \mathrm{~mm}, \mathrm{w}=0.5 \mathrm{~mm}$ are selected, then $\mathrm{y}$ is changed. Figure 8 shows $\mathrm{S} 41$ under different $\mathrm{y}$. The conclusions are obtained from figure 8 that distance from left edges of the cavity to microstrip line in horizontal has little impact on reducing far-end crosstalk. The effect of reducing far-end crosstalk is nonlinear increasing with increasing of cavity width, combined with figure 7 . When we compare the figure 8 and figure 3,the conclusions are found that the effect of reducing far-end crosstalk is similar in the structure of taking no any method and the structure of $\mathrm{y}=0.6 \mathrm{~mm}$, $\mathrm{y}=0.5 \mathrm{~mm}, \mathrm{y}=0.4 \mathrm{~mm}$.

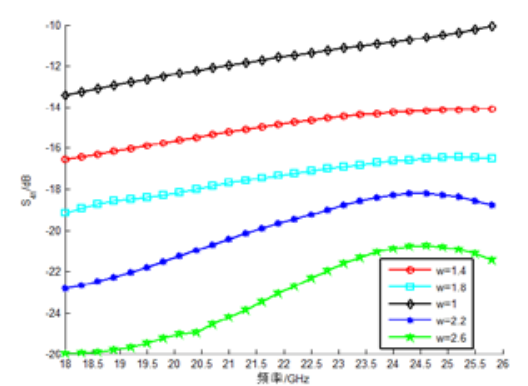

Fig. $7 \mathrm{~S}_{41}$ under different $\mathrm{w}$

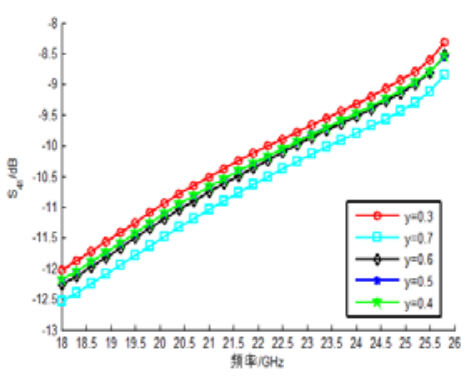

Fig. $8 \mathrm{~S}_{41}$ under different $\mathrm{y}$

\section{Conclusions}

The paper gets the following several important conclusions by analyzing and summarizing:

(1)Among structural parameters installed of the cavity, it is a optimal structure for reducing far-end crosstalk from $18 \mathrm{GHz}$ to $25.8 \mathrm{GHz}$ that $\mathrm{x}=1 \mathrm{~mm}, \mathrm{y}=1 \mathrm{~mm}, \mathrm{z}=0.06, \mathrm{l}=8 \mathrm{~mm}, \mathrm{w}=3, \mathrm{~h}$ $=0.14$. Far-end crosstalk is reduced more than $30 \mathrm{~dB}$.

(2)The effect of reducing far-end crosstalk is nonlinear increasing with increasing of cavity width, the effect is increasing and then decreasing with increasing of cavity height. Cavity height of effect best was $0.14 \mathrm{~mm}$. When location of sample cavity in dielectric layer was different, the effect was different, distance from bottom of cavity to ground plane of the effect best is $0.06 \mathrm{~mm}$.

\section{Acknowledgements}

This work was supported by advanced research project “The Study of *** Multi-energy***” .

\section{Reference}

[1] Xiong Q S, Wu Z H, Chen P, et al. Analysis of Characteristic of Microstrip Signal Loss in Course of Signal Transmission[J].Advanced Materials Research, 2011194-196:2229-2232.

[2] Xu Xin. Analysis and modeling of transitions of millimeter-wave transmission lines of LTCC 
and parasitic mode[D].University of electronic science and technology, 2009.

[3] An Jing, WU Junfeng, WU Yihui, et al. Influence of the Structural Parameters of Via Fences on Reducing the Crosstalk Between Coupled Microstrip Lines[J].Journal Of Wuhan University Of Technology, 2010 (23) : 81-84.

[4] LEE K, LEE H B, JUNG H K, et al. A Serprntine guard trace to reduce the far-end crosstalk voltage and the crosstalk induced timing jitter of parallel microstrip lines[J].IEEE Trans on Advanced Packaging, 2008, 31( 4) : 809-817.

[5] Lin Ding-bing, Wang Chen kuang and LU Chi hao, et al., Using rectangular shape - resonators to improve the far-end crosstalk of the coupled microstrip lines[C]//Proc of PIERS. 2011:1612-1616.

[6] Sun Xuyin, Lin Zhili, Ou Pan, et al. Study of far-end crosstalk between microstrip lines with electromagnetic simulation[J].Application research of Computers, 2014 (9) : 2665-2667.

[7] Maric A M, Radosavljevic G J, Smetana W, et al.Com parison of LTCC inductors on company's substrate configurations with PCB inductor[J].Journal of Microelectronics International, 2013, 31 (1) : 32-41 (10).

[8] Elsherbeni, Atef Z, Demir, Veysel, et al. The finite-difference time-domain method for electromagnetics with MATLAB simulation[J] [S.I.] : SciTech Publishing Inc, 2009. 